\title{
Statistical error estimates in dynamical mean-field theory and extensions thereof
}

\author{
Patrick Kappl, Markus Wallerberger, Josef Kaufmann, Matthias Pickem, Karsten Held \\ Institute of Solid State Physics, TU Wien, 1040 Vienna, Austria
}

(Dated: August 14, 2020)

\begin{abstract}
We employ the jackknife algorithm to analyze the propagation of the statistical quantum Monte Carlo error through the Bethe-Salpeter equation. This allows us to estimate the error of dynamical mean-field theory calculations of the susceptibility and of dynamical vertex approximation calculations of the self-energy. We find that the different frequency components of the susceptibility are uncorrelated, whereas those of the self-energy are correlated. For improving the quality of the correlation matrix taking sufficiently many jackknife bins is key, while for reducing the standard error of the mean sufficiently many Monte Carlo measurements are necessary. We furthermore show that even in the case of the self-energy, the finite covariance does not have a sizable influence on the analytic continuation.
\end{abstract}

\section{INTRODUCTION}

Developing reliable theories for strong electronic correlation has proved a Herculean task. Three decades after its invention [1] 3], dynamical mean-field theory (DMFT) has become state-of-the-art to calculate strongly correlated models 4 and materials [5]9]. Notwithstanding, one of the core scientific tasks, namely providing a proper error estimate for such calculations, is still in its infancy. Error estimates which directly follow from the quantum Monte Carlo (QMC) simulation of the (self-consistently determined) DMFT impurity problem, e.g. for the magnetization or compressibility, have been provided already from the beginning of DMFT, see, e.g., Refs. 3, 10, and 11. But as the focus of such calculations is nowadays more on the one-particle spectral function or two-particle susceptibility, error estimates are by and large missing.

This is because such an error estimate is nontrivial. First, calculating the spectrum or susceptibility requires complex, non-linear post-processing routines such as a maximum entropy analytic continuation or the BetheSalpeter equation, respectively. Besides proper error propagation through these post-processing procedures, there is, secondly, the iteration error, i.e., the difference between the numerical solution and the exact (true) DMFT fixed point. Third, DMFT is an approximation to the correlation problem itself, introducing a systematic error for finite dimensional systems.

The latter, i.e., the error of DMFT as an approximation, can be systematically improved upon by either cluster 12 or diagrammatic extensions 13. Cluster extensions replace the single impurity site with a small cluster, thus interpolating between DMFT and the full lattice problem. Given proper finite size scaling, enlarging the cluster then yields an error estimate. The huge numerical effort essentially restricts such cluster extensions however to small clusters on one- and two-dimensional lattices.

Diagrammatic extensions, on the other hand, augment DMFT with a specific set of non-local Feynman diagrams. The dynamical vertex approximation (DГA) [14, 15], a prominent diagrammatic extension, generalizes the concept of a local one-particle vertex (selfenergy) in DMFT systematically to the two-, three-, $n$ - particle vertex. For $n \rightarrow \infty$ one recovers the full problem. The corrections on the two-particle vertex level provide an error estimate for the DMFT approximation, and similarly those of the three-particle level as an error estimate for the ВГА results on the two-particle vertex level. One can proceed in a similar fashion of approximating the error [16] in the dual Fermion approach[17]. Since both the size of the vertices and the effort of the associated diagrammatic equations grow strongly in $n$, one is restricted to small $n$.

In this paper, we focus on the error propagation through the post-processing procedure, specifically, the Bethe-Salpeter equation. This does not only involve the DMFT calculation of the susceptibility but also the DГA calculation of the self-energy which employs the same Bethe-Salpeter equation, and on top of that the Schwinger-Dyson equation. Input for both equations is the local two-particle Green's function and the properly converged DMFT one-particle Green's function. For multi-orbital systems or at low temperatures the twoparticle Green's function is only accessible using QMC techniques, but requires quite some effort to calculate and hence has a substantial statistical QMC error. We employ the jackknife method [18, 19] to analyze the propagation of the QMC error through the Bethe-Salpeter equation and to estimate the error of the final DMFT susceptibility and DГA self-energy. Finally, we perform maximum entropy analytic continuations of the DГA selfenergy, using the jackknife estimates for error and covariance.

The remainder of the paper is organized as follows: Section [II describes the methods employed: DMFT, continuous-time quantum Monte Carlo, AbinitioDГA, and jackknife resampling. The input to our calculations, the two-particle Green's function, is analyzed in Section III. The main results for the DГA self-energy and the DMFT susceptibilities are presented in Sections IV and $\mathrm{V}$ respectively, while an analytical continuation of the DГA self-energy is done in Section VI A discussion and conclusion can be found in Section VII. 


\section{METHODS}

In the following, we briefly recapitulate the essential steps for calculating the DMFT susceptibility and DГA self-energy, starting from the QMC calculation of the oneand two-particle Green's function. We restrict ourselves to the essential equations without discussing technical details such as reformulations of the equations or numerical efficiency. For these details we refer the reader to Ref. 20 as regards the QMC calculation with the w2dynamics package [20] and to Ref. 21] for a general review, to Refs. 22 and 23 as regards the calculation of the DMFT susceptibility and DГA self-energy with the $a b$ initio $d y$ namical vertex approximation (ADGA) package, and to Ref. 13 for a review. Further, we discuss the essential idea of the jackknife algorithm, again referring the reader to the review Ref. 19 for a more detailed presentation.

\section{A. Dynamical mean-field theory}

In DMFT, we map the lattice model

$$
H_{\text {lattice }}=U \sum_{i} \hat{c}_{i \uparrow}^{\dagger} \hat{c}_{i \downarrow}^{\dagger} \hat{c}_{i \downarrow} \hat{c}_{i \uparrow}+\sum_{\sigma, i, j} t_{i j} \hat{c}_{i \sigma}^{\dagger} \hat{c}_{j \sigma},
$$

where $\hat{c}_{i \sigma}$ annihilates a fermion of spin $\sigma$ on site $i, U$ is the on-site interaction, and $t_{i j}$ is the hopping matrix, onto an Anderson impurity model (AIM) 2, 3]:

$$
\begin{aligned}
H_{\mathrm{AIM}} & =U \hat{c}_{\uparrow}^{\dagger} \hat{c}_{\downarrow}^{\dagger} \hat{c}_{\downarrow} \hat{c}_{\uparrow}+\sum_{\sigma} \tilde{\epsilon}_{\sigma} \hat{c}_{\sigma}^{\dagger} \hat{c}_{\sigma} \\
& +\underbrace{\sum_{\sigma, p}\left(V_{p} \hat{c}_{\sigma}^{\dagger} \hat{f}_{\sigma p}+V_{p}^{*} \hat{f}_{\sigma p}^{\dagger} \hat{c}_{\sigma}\right)}_{H_{\mathrm{hyb}}}+\sum_{p} \epsilon_{p} \hat{f}_{\sigma p}^{\dagger} \hat{f}_{\sigma p} .
\end{aligned}
$$

Here $U$ is the impurity interaction which is the same as that of the original lattice problem; $V_{p}$ denotes the hybridization between the impurity (denoted by $\hat{c}_{\sigma}^{\dagger}$ and $\hat{c}_{\sigma}$ creation and annihilation operators for spin $\sigma \in\{\uparrow, \downarrow\})$ and bath site $p$ (denoted by corresponding $\hat{f}_{\sigma p}^{\dagger}$ and $\hat{f}_{\sigma p}$ operators) at energy $\epsilon_{p}$. In essence, DMFT determines an AIM that gives the same local one-particle physics as the lattice model where the corresponding parameters $V_{p}$ and $\epsilon_{p}$ (or the hybridization function $\Delta$ ) have to be determined self-consistently [2, 3]. In what follows we assume that this DMFT self-consistency has been achieved to high accuracy. For the sake of simplicity, we have restricted the equations to the one-orbital problem, but the generalization to multi-orbital models is straightforward.

\section{B. Continuous-time quantum Monte Carlo}

In order to obtain the one- and two-particle Green's function for the Anderson impurity model (2), we use continuous-time quantum Monte Carlo in the hybridization expansion (CT-HYB) 21, 24] with worm sampling 25] as implemented in the w2dynamics package 20, 26]. CT-HYB with worm sampling proceeds in a three-step fashion: First, one splits the Hamiltonian $H$ into an interacting part, taken to be $H_{I}=H_{\text {hyb }}$, and the rest, $H_{0}$. Second, one expands both the partition function and the expectation value of some observable ("worm" $\mathcal{W}$ ) into a Dyson series with respect to $H_{I}$ and uses Wick's theorem to group diagrams into determinants. For the partition functions, this yields:

$$
\begin{aligned}
\mathcal{Z} & =\sum_{n=0}^{\infty} \frac{(-1)^{n}}{n !} \sum_{\sigma_{1}, \sigma_{1}^{\prime}} \cdots \sum_{\sigma_{n}, \sigma_{n}^{\prime}} \int_{0}^{\beta} \mathrm{d}^{\mathrm{n}} \tau \mathrm{d}^{\mathrm{n}} \tau^{\prime} \\
& \times \operatorname{Tr}\left[T_{\tau} \mathrm{e}^{-\beta H_{\mathrm{loc}}} \prod_{i=1}^{n} \hat{c}_{\sigma_{i}}^{\dagger}\left(\tau_{i}\right) \hat{c}_{\sigma_{i}^{\prime}}\left(\tau_{i}^{\prime}\right)\right] \operatorname{det} \boldsymbol{\Delta}
\end{aligned}
$$

where $T_{\tau}$ denotes time ordering. The elements of the hybridization matrix $\boldsymbol{\Delta}$ are given by $\boldsymbol{\Delta}_{i j}=$ $\Delta_{\sigma_{i} \sigma_{j}^{\prime}}\left(\tau_{i}-\tau_{j}^{\prime}\right)$ with the hybridization function $\Delta_{\sigma \sigma^{\prime}}(\tau)=$ $\delta_{\sigma \sigma^{\prime}} \sum_{p} V_{p}\left(\partial_{\tau}-\epsilon_{p}\right)^{-1} V_{p}^{*}$.

Similarly, we write down the hybridization expansion for the worm operator $\mathcal{W}(\{\tilde{\tau}\})$. It can consist of several creation and annihilation operators with various number of time arguments. Most important examples are the one- and two-particle Green's function, where $\mathcal{W}(\{\tilde{\tau}\})$ stands for $T_{\tau} \hat{c}_{\sigma}(\tau) \hat{c}_{\sigma}^{\dagger}\left(\tau^{\prime}\right)$ and $T_{\tau} \hat{c}_{\sigma}\left(\tau_{1}\right) \hat{c}_{\sigma}^{\dagger}\left(\tau_{2}\right) \hat{c}_{\sigma}\left(\tau_{3}\right) \hat{c}_{\sigma}^{\dagger}\left(\tau_{4}\right)$, respectively. Other worm operators have been introduced in Refs. 27 29, For the sampling space of $\mathcal{W}(\{\tilde{\tau}\})$, we thus get:

$$
\begin{aligned}
\mathcal{Z}_{\mathcal{W}} & =\sum_{n=0}^{\infty} \frac{(-1)^{n}}{n !} \sum_{\sigma_{1}, \sigma_{1}^{\prime}} \cdots \sum_{\sigma_{n}, \sigma_{n}^{\prime}} \int_{0}^{\beta} \mathrm{d}^{\mathrm{n}} \tau \mathrm{d}^{\mathrm{n}} \tau^{\prime} \mathrm{d}\{\tilde{\tau}\} \\
& \times \operatorname{Tr}\left[T_{\tau} \mathrm{e}^{-\beta H_{\mathrm{loc}}} \mathcal{W}(\{\tilde{\tau}\}) \prod_{i=1}^{n} \hat{c}_{\sigma_{i}^{\dagger}}\left(\tau_{i}\right) \hat{c}_{\sigma_{i}^{\prime}}\left(\tau_{i}^{\prime}\right)\right] \operatorname{det} \boldsymbol{\Delta} .
\end{aligned}
$$

Third, we combine both sampling spaces by taking the abstract sum $\mathcal{Z}+\eta \mathcal{Z}_{\mathcal{W}}$, where $\eta$ is a balancing parameter. The resulting space is sampled using Markov chain Monte Carlo.

An estimator for the worm operator $\mathcal{W}(\{\tilde{\tau}\})$ is then simply given by:

$$
\langle\mathcal{W}(\{\tilde{\tau}\})\rangle=\frac{\mathcal{Z}_{\mathcal{W}}}{\mathcal{Z}}\langle\sigma(\{\tilde{\tau}\})\rangle,
$$

where $\sigma\left(\left\{\tau_{i}\right\}\right)$ is the indicator function of a configuration in $\mathcal{Z}_{\mathcal{W}}$ with the matching times, $\mathcal{Z}_{\mathcal{W}} / \mathcal{Z}$ is the ratio of volumes between the two spaces. Let us note that in the case of the one- or two-particle Green's function one worm measurement is computationally cheaper than one measurement of the removal estimator in $Z$-sampling, but it also yields less information. 


\section{DMFT susceptibility}

In the following, we will make the transition from imaginary time to Matsubara frequencies, where the oneparticle Green's function is

$$
G_{\sigma}^{\nu}=\int_{0}^{\beta} \mathrm{d} \tau e^{i \nu(\tau)}\left\langle T_{\tau} \hat{c}_{\sigma}(\tau) \hat{c}_{\sigma}^{\dagger}(0)\right\rangle
$$

and the two-particle Green's function is

$$
\begin{array}{r}
G_{\sigma \sigma^{\prime}}^{(2), \nu \nu^{\prime} \omega}=\frac{1}{\beta} \int_{0}^{\beta} \mathrm{d} \tau_{1} \mathrm{~d} \tau_{2} \mathrm{~d} \tau_{3} e^{i\left[\nu \tau_{1}-(\nu+\omega) \tau_{2}+\left(\nu^{\prime}+\omega\right) \tau_{3}\right]} \\
\left\langle T_{\tau} \hat{c}_{\sigma}\left(\tau_{1}\right) \hat{c}_{\sigma}^{\dagger}\left(\tau_{2}\right) \hat{c}_{\sigma^{\prime}}\left(\tau_{3}\right) \hat{c}_{\sigma^{\prime}}^{\dagger}(0)\right\rangle .
\end{array}
$$

Note that here and in the following we use the notation of the w2dynamics code [20] which has the extra $1 / \beta$ factor in the definition of the two-particle Green's function (7) compared to Ref. 13. From these Green's functions, we can calculate the generalized local susceptibility

$$
\chi_{\sigma \sigma^{\prime}}^{\nu \nu^{\prime} \omega}=\beta G_{\sigma \sigma^{\prime}}^{(2), \nu \nu^{\prime} \omega}-\beta G_{\nu} G_{\nu^{\prime}} \delta_{\omega 0} .
$$

Diagrammatically, the above equation means that we remove one disconnected contribution from $G^{(2)}$. In the presence of $\mathrm{SU}(2)$ symmetry, i.e., in the paramagnetic phase, one can further restrict oneself to the following two spin combinations, also referred to as density $(d)$ and magnetic $(m)$ channel.

$$
\chi_{d / m,}^{\nu \nu^{\prime} \omega}=\chi_{\uparrow \uparrow}^{\nu \nu^{\prime} \omega} \pm \chi_{\uparrow \downarrow}^{\nu \nu^{\prime} \omega} .
$$

From the local susceptibility $\chi_{r}^{\nu \nu^{\prime} \omega}$ with $r \in\{d, m\}$ and the local bare bubble susceptibility $\chi_{0}^{\nu \nu^{\prime} \omega}=$ $-\beta G_{\nu} G_{(\nu+\omega)} \delta_{\nu \nu^{\prime}}$, we can determine the local irreducible vertex through an inversion of the local Bethe-Salpeter equation

$$
\Gamma_{r}=\chi_{r}^{-1}-\chi_{0}^{-1} .
$$

This equation is diagonal with respect to each bosonic frequency $\omega$ while the inversion itself is performed in the implicit matrix notation $\nu$ and $\nu^{\prime}$. From $\Gamma$ and the local particle-hole bubble $\chi_{0, \mathbf{k} \mathbf{k}^{\prime} \mathbf{q}}^{\nu{ }^{\prime} \omega}=$ $-\beta G_{\mathbf{k} \nu} G_{(\mathbf{k}+\mathbf{q})(\nu+\omega)} \delta_{\nu \nu^{\prime}} \delta_{\mathbf{k k}^{\prime}}$ the lattice Bethe-Salpeter equation now allows us to calculate the generalized DMFT lattice susceptibility

$$
\chi_{r, \mathbf{k} \mathbf{k}^{\prime} \mathbf{q}}^{\nu \nu^{\prime} \omega}=\chi_{0, \mathbf{k} \mathbf{k}^{\prime} \mathbf{q}}^{\nu \nu^{\prime} \omega}-\sum_{\substack{\nu_{1} \nu_{2} \\ \mathbf{k}_{1} \mathbf{k}_{\mathbf{2}}}} \chi_{0, \mathbf{k} \mathbf{k}_{1} \mathbf{q}}^{\nu \nu_{1} \omega} \Gamma_{r}^{\nu_{1} \nu_{2} \omega} \chi_{r, \mathbf{k}_{\mathbf{2}} \mathbf{k}^{\prime} \mathbf{q}}^{\nu_{2} \nu^{\prime} \omega}
$$

Here and in the following, we distinguish lattice and local quantities through the additional momentum indices $\mathbf{k}$, $\mathbf{k}^{\prime}$, $\mathbf{q}$; and implicitly assume a factor $1 / \beta$ for each Matsubara frequency sum, as in Ref. 13. That is, $\sum_{\nu_{1}}$ actually means $\frac{1}{\beta} \sum_{\nu_{1}}$. From these, physical susceptibilities at frequency $\omega$ and momentum q can be calculated through summing over $\nu, \nu^{\prime}, \mathbf{k}, \mathbf{k}^{\prime}$.

\section{AbinitioDГA self-energy}

Similar to Eq. 111), we can also calculate the full vertex $F$ through the Bethe-Salpeter equation

$$
F_{r, \mathbf{k k}^{\prime} \mathbf{q}}^{\nu \nu^{\prime} \omega}=\Gamma_{r}^{\nu \nu^{\prime} \omega}+\sum_{\substack{\nu_{1} \nu_{2} \\ \mathbf{k}_{1} \mathbf{k}_{2}}} \Gamma_{r}^{\nu \nu_{1} \omega} \chi_{0, \mathbf{k}_{\mathbf{1}} \mathbf{k}_{\mathbf{2}} \mathbf{q}}^{\nu_{1} \nu_{2} \omega} F_{r, \mathbf{k}_{\mathbf{2}} \mathbf{k}^{\prime} \mathbf{q}}^{\nu_{2} \nu^{\prime} \omega}
$$

However in the ladder approximation the resulting $F$ from Equation (12) simplifies further and does not have an explicit dependence on $\mathbf{k}$ and $\mathbf{k}^{\prime}$ :

$$
F_{r, \mathbf{q}}^{\nu \nu^{\prime} \omega}=\Gamma_{r}^{\nu \nu^{\prime} \omega}+\sum_{\nu_{1} \nu_{2}} \Gamma_{r}^{\nu \nu_{1} \omega} \chi_{0, \mathbf{q}}^{\nu_{1} \nu_{2} \omega} F_{r, \mathbf{q}}^{\nu_{2} \nu^{\prime} \omega} .
$$

Here we defined $\chi_{0, \mathbf{q}}^{\nu \nu^{\prime} \omega}=\sum_{\mathbf{k}, \mathbf{k}^{\prime}} \chi_{0, \mathbf{k} \mathbf{k}^{\prime} \mathbf{q}}^{\nu \nu^{\prime} \omega}$. Please note that the generated non-local full vertices $F$ in Equation (13) are no longer crossing symmetric. By taking into account the corresponding diagrams in the transversal particlehole channel we get for the density component

$$
\begin{aligned}
F_{d, \mathbf{k} \mathbf{k}^{\prime} \mathbf{q}}^{\nu \nu^{\prime} \omega}=F_{d, \mathbf{q}}^{\nu \nu^{\prime} \omega} & +\frac{1}{2} F_{d, \mathbf{k}^{\prime}-\mathbf{k}}^{\mathrm{nl}\left(\nu^{\prime}-\nu\right)\left(\nu^{\prime}-\omega\right) \nu^{\prime}} \\
& +\frac{3}{2} F_{m, \mathbf{k}^{\prime}-\mathbf{k}}^{\mathrm{nl}\left(\nu^{\prime}-\nu\right)\left(\nu^{\prime}-\omega\right) \nu^{\prime}}
\end{aligned}
$$

where we defined $F_{r, \mathbf{q}}^{\mathrm{nl} \nu \nu^{\prime} \omega}=F_{r, \mathbf{q}}^{\nu \nu^{\prime} \omega}-F_{r}^{\nu \nu^{\prime} \omega}$. From this vertex we can calculate the DГA self-energy

$$
\begin{gathered}
\Sigma_{\mathbf{k} \nu}=\frac{U n}{2}-U \sum_{\substack{\nu^{\prime} \omega \\
\mathbf{k}^{\prime} \mathbf{q}}} F_{d, \mathbf{k} \mathbf{k}^{\prime} \mathbf{q}}^{\nu \nu^{\prime}} G_{\mathbf{k}^{\prime} \nu^{\prime}} G_{\left(\mathbf{k}^{\prime}+\mathbf{q}\right)\left(\nu^{\prime}+\omega\right)} \\
\times G_{(\mathbf{k}+\mathbf{q})(\nu+\omega)},
\end{gathered}
$$

where $n$ is the electron density entering in the Hartree term. The actual calculations for both the DMFT susceptibility and the DГA self-energy are done using the ADGA program package which together with further computational details is discussed in Refs. 22 and 23

\section{E. Jackknife}

The jackknife is a resampling method, used for bias reduction and error estimation. It is a versatile method for a range of problems, hence the name [18, 19.

Before we describe the jackknife in detail, let us recapitulate some statistical terms that we will need later on. Let $\theta$ be the true, yet unknown, value of some quantity. To access $\theta$ we construct an estimator, $\hat{\theta}$, which is a function of $n$ random variables, $X_{1}, \ldots, X_{n}$. In our case $X_{i}$ are just Monte Carlo measurements. The bias of the estimator $\hat{\theta}$ is then given by the difference between its expectation value $\mathrm{E}[\hat{\theta}]$ and the true value $\theta$,

$$
\operatorname{bias}[\hat{\theta}]:=\mathrm{E}[\hat{\theta}]-\theta .
$$

For $\hat{\theta}$ to be useful, its bias must be systematically improvable by increasing the sample size $n$. More specifically, 
a sequence of estimators $\left\{\hat{\theta}_{n}\left(X_{1}, \ldots, X_{n}\right)\right\}$ is called consistent with $\theta$ if and only if the probability of deviating from $\theta$ goes to zero with $n$, i.e.,

$$
\lim _{n \rightarrow \infty} \operatorname{Pr}\left(\left|\hat{\theta}_{n}\left(X_{1}, \ldots, X_{n}\right)-\theta\right|>\epsilon\right)=0, \quad \forall \epsilon>0 .
$$

Now let us explain jackknife resampling based on the following general problem. Assume we want to calculate some quantity $y=f(x)$, where $f$ is some arbitrary, known function. If we knew the true value of $x$ the task would be trivial. In our case, however, $x$ is a random variable and we only have access to a sample $\left\{x_{i}\right\}$ of size $n$. Therefore we need to find a good estimator $\hat{y}$ and be able to quantify its error. In Sections [V] and $\mathrm{V}$ the input samples $x_{i}$ are the two-particle Green's functions from QMC calculations, $y$ is either the DГA self-energy or the DMFT susceptibility, $f$ is given by the BetheSalpeter equation and in case of the self-energy also by the Schwinger-Dyson equation.

For simplicity, we only propagate the error in the twoparticle Green's function. This is justified, because the one-particle Green's function is calculated by symmetric improved estimators and thus its error is smaller by several orders of magnitude 29 .

The general strategy of resampling techniques is to generate subsamples from the initial one which are preferably independent and identically distributed (iid). With this, one can then estimate certain statistics of the sample like its mean or variance. In case of the jackknife a bias estimation and reduction is also possible. To show this, let $\hat{y}_{n}$ be a consistent estimator function for $y$. A common choice that we used for all calculations in this paper is

$$
\hat{y}_{n}\left(x_{1}, \ldots, x_{n}\right)=f(\bar{x}),
$$

where $f$ is the function from the original problem and $\bar{x}$ is the input sample mean. The latter is an unbiased, consistent estimator for $x$. If $f$ is a continuous function, it is easy to see that $f(\bar{x})$ is a consistent estimator for $y$. We note that if $f$ is linear, then $f(\bar{x})$ is already unbiased.

After choosing $\hat{y}_{n}$ we generate $n$ leave-one-out samples

$$
y_{-i}=\hat{y}_{n-1}\left(x_{1}, \ldots, x_{i-1}, x_{i+1}, \ldots, x_{n}\right) \text {. }
$$

Note that this simple choice of resampling implies that the leave-one-out samples are also consistent estimators for $y$ and carry a different but similar bias. The reason why this is important for obtaining a bias-corrected estimator will become clearer in the following. Consistency requires the bias to vanish in the limit of $n \rightarrow \infty$. Thus, if $f$ is not only continuous but also analytic around the true value $x$, we can expand the bias of $\hat{y}_{n}$ in powers of $1 / n$ and write

$$
\begin{aligned}
\operatorname{bias}\left[\hat{y}_{n}\right] & =\frac{a}{n}+\frac{b}{n^{2}}+O\left(n^{-3}\right), \\
\operatorname{bias}\left[y_{-i}\right] & =\operatorname{bias}\left[\hat{y}_{n-1}\right]=\frac{a}{n-1}+\frac{b}{(n-1)^{2}}+O\left(n^{-3}\right),
\end{aligned}
$$

with some $a$ and $b$. The expectation value as well as the bias are linear operators [see Eq. (16)]. It is therefore easy to construct new samples $y_{i}^{\prime}$ in such a way that we get rid of the leading $O(1 / n)$ term in the bias

$$
\begin{aligned}
y_{i}^{\prime} & =n \hat{y}_{n}-(n-1) y_{-i}, \\
\operatorname{bias}\left[y_{i}^{\prime}\right] & =n \operatorname{bias}\left[\hat{y}_{n}\right]-(n-1) \operatorname{bias}\left[y_{-i}\right] \\
& =\left(a+\frac{b}{n}\right)-\left(a+\frac{b}{n-1}\right)+O\left(n^{-3}\right) \\
& =-\frac{b}{n(n-1)}+O\left(n^{-3}\right)=O\left(n^{-2}\right) .
\end{aligned}
$$

By calculating the sample mean of the $y_{i}^{\prime}$ we finally arrive at the bias-corrected jackknife estimator

$$
\hat{y}_{\mathrm{JK}}=\bar{y}^{\prime}=\frac{1}{n} \sum_{i=1}^{n} y_{i}^{\prime} .
$$

Its expectation value is given by

$$
\begin{aligned}
\mathrm{E}\left[\hat{y}_{\mathrm{JK}}\right] & =\frac{1}{n} \sum_{i=1}^{n}\left(\mathrm{E}\left[y_{i}^{\prime}\right]\right)=\frac{1}{n} \sum_{i=1}^{n}\left(n \mathrm{E}\left[\hat{y}_{n}\right]-(n-1) \mathrm{E}\left[y_{-i}\right]\right) \\
& =\frac{1}{n} \sum_{i=1}^{n}\left(n\left(y+\operatorname{bias}\left[\hat{y}_{n}\right]\right)-(n-1)\left(y+\operatorname{bias}\left[y_{-i}\right]\right)\right) \\
& =\frac{1}{n} \sum_{i=1}^{n}\left(y+\operatorname{bias}\left[y_{i}^{\prime}\right]\right)=y+O\left(n^{-2}\right),
\end{aligned}
$$

where Eq. 23 was used in the last step. This shows that $\hat{y}_{\mathrm{JK}}$ is a consistent estimator for $y$ with a reduced bias of $O\left(1 / n^{2}\right)$.

Without proof, consistent estimators for the variance, standard deviation, covariance, etc. of $y$ can also be obtained by calculating the corresponding sample estimates of $\left\{y_{i}^{\prime}\right\}[30$. In Sections III to V we are specifically interested in the standard error of the mean (SEM) and the linear correlation, $\operatorname{corr}[\cdot, \cdot]$. The latter is estimated by the sample Pearson correlation coefficients $r$. For scalar random variables $p$ and $q$ with samples $\left\{p_{i}\right\}$ and $\left\{q_{i}\right\}$ of size $n$ the following holds

$$
\begin{aligned}
\operatorname{SEM}_{p} & :=\frac{s_{p}}{\sqrt{n}}=\sqrt{\frac{1}{n(n-1)} \sum_{i=1}^{n}\left|p_{i}-\bar{p}\right|^{2}} \\
\widehat{\operatorname{corr}}[p, q] & =r_{p q}=\frac{\sum_{i=1}^{n}\left(p_{i}-\bar{p}\right)\left(q_{i}-\bar{q}\right)^{*}}{\sqrt{\sum_{i=1}^{n}\left|p_{i}-\bar{p}\right|^{2}} \sqrt{\sum_{i=1}^{n}\left|q_{i}-\bar{q}\right|^{2}}} .
\end{aligned}
$$

Here $s$ denotes the corrected sample standard deviation, $\bar{p}$ the sample mean of the $p_{i}$, and $\widehat{\text { corr }}$ the estimated correlation. The generalization to random vectors and objects of higher rank is straightforward by componentwise application of the above formulas. In the following, $p_{i}$ is a scalar component of the $i$ th sample $x_{i}$ or $y_{i}$, e.g., 
$p_{i}=\left(\Sigma_{\mathrm{D} \Gamma \mathrm{A}, \mathbf{k}=(0,0), \nu=\pi / \beta}\right)_{i}$ might be the $i$ th measurement of the self-energy at a fixed momentum $\mathbf{k}$ and frequency $\nu$.

Let us note an important caveat in using Eq. (27) as estimator for a $k \times k$ correlation matrix $r_{p q}$ with a large number of features $k$ : While each component of the covariance converges as $1 / \sqrt{n}$ regardless of $k$, the eigenvalues of the covariance matrix, which are used to construct independent errors, converge only as a function of $n / k$. In particular, the estimator yields a singular correlation matrix for any $n<k$.

For practical use, the whole derivation and discussion of the jackknife above can be condensed into three simple steps:

\section{Resample}

$$
x_{i} \rightarrow x_{i}^{\prime}=\frac{1}{n-1} \sum_{j \neq i} x_{j}=\frac{n \bar{x}-x_{i}}{n-1}
$$

\section{Transform}

$$
y_{i}^{\prime}=n f(\bar{x})-(n-1) f\left(x_{i}^{\prime}\right)
$$

3. Calculate sample statistics of $\left\{y_{i}^{\prime}\right\}$, e.g., Eqs. 24, (26) and (27)

Another statistical method, similar to the jackknife, is the bootstrap. It is more powerful but usually requires a greater number of resamples to take advantage of that 31. Depending on the specific problem, at least hundreds or thousands of new samples are drawn for the bootstrap method 30, 32] as opposed to the 16 to 256 jackknife samples that are used in Sections IV and $\mathrm{V}$ Since each resample requires a full DГA calculation, the jackknife is computationally cheaper and therefore the method of choice.

\section{F. Parallel implementation}

The main focus of this paper is on the jackknife estimates of the self-energy and susceptibilities calculated within ADGA. In this case the parallelization is simple, because the ADGA calculation is by far the most computationally intensive task and already implemented in a parallel way. Therefore the actual jackknife part is programmed in serial and only the calls to the ADGA code are done in parallel.

\section{STATISTICAL ANALYSIS OF THE INPUT: TWO-PARTICLE GREEN'S FUNCTION}

Before we analyze the DГA self-energy and DMFT susceptibilities, let us take a closer look at the input of the DMFT and DГA calculations, namely the twoparticle Green's function $G^{(2), \nu \nu^{\prime} \omega}$. In particular we want
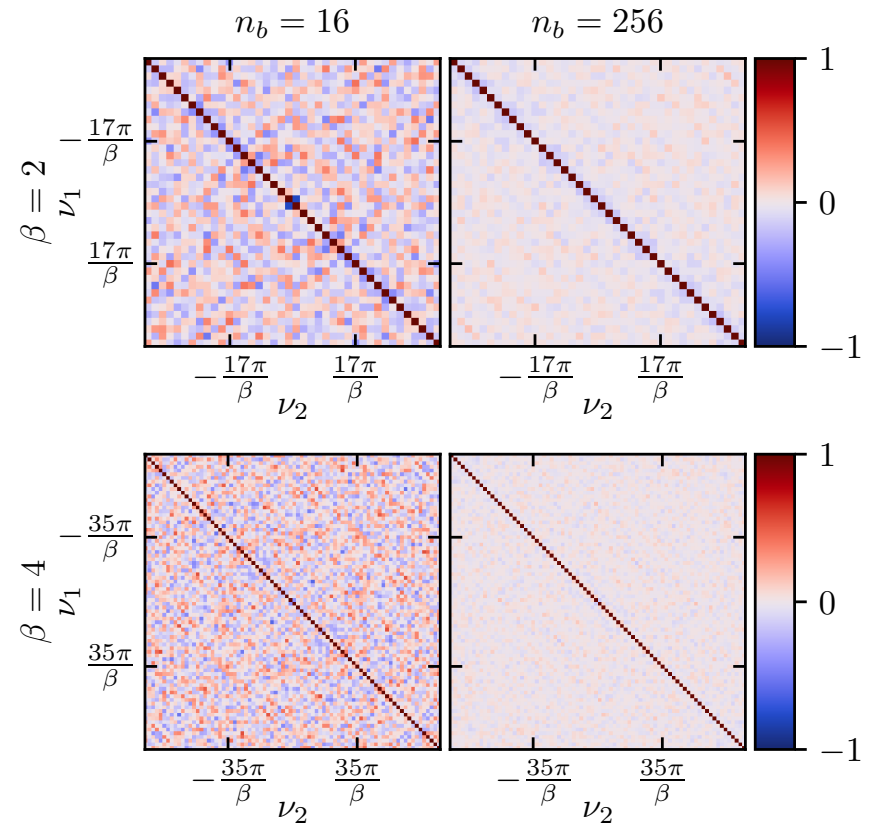

Figure 1. Estimated correlation matrix $\widehat{\operatorname{corr}}\left[G_{\text {cut1 }}^{(2)}\left(\nu_{1}\right), G_{\text {cut1 }}^{(2)}\left(\nu_{2}\right)\right]$ for the two-particle Green's function at the cut $\nu=\nu^{\prime}=\nu_{i}, \omega=0$ comparing different temperatures and numbers of bins. The $40 \times 40$ matrices in the top row and the $80 \times 80$ matrices in the bottom row correspond to 40 and 80 fermionic frequencies, respectively.

to check if the correlations of the self-energy and susceptibilities are completely intrinsic or if they originate at least in part from the input. For this reason we estimate $\operatorname{corr}\left[G_{\uparrow \uparrow}^{(2), \nu_{1} \nu_{1}^{\prime} \omega_{1}}, G_{\uparrow \uparrow}^{(2), \nu_{2} \nu_{2}^{\prime} \omega_{2}}\right]$ for various frequency combinations, and plot two-dimensional cuts of this highdimensional quantity.

All QMC simulations were done for the 2D square lattice Hubbard model at half-filling using the following parameters: $U=4 t, \beta=\{2 / t, 4 / t\}$, where the hopping amplitude $t=1$ serves as our energy unit. The hopping matrix in Equation (1) is taken to permit only nearestneighbor hopping. The number of fermionic frequencies is 40 for $\beta=2$ and 80 for $\beta=4$. Due to the imposed particle-hole symmetry, the two-particle Green's function and therefore also its correlation matrix is purely real. Before the latter was estimated, the total number of $n_{t}$ QMC measurements were divided equally into $n_{b}$ bins. The $n_{m}=n_{t} / n_{b}$ measurements in each bin were then averaged and used as the samples for the estimations. All results in this section were obtained with $n_{m}=2.4 \times 10^{6}$.

In Fig. 1 1 the estimated correlation of

$$
G_{\mathrm{cut} 1}^{(2)}\left(\nu_{1}\right):=G_{\uparrow \uparrow}^{(2), \nu=\nu_{1}, \nu^{\prime}=\nu_{1}, \omega=0}
$$

with itself is shown for two temperatures $\beta=2$ (top) and $\beta=4$ (bottom) and two numbers of bins $n_{b}=16$ (left) and $n_{b}=256$ (right). Although there is quite some noise in the plots with fewer bins, the diagonal matrix structure is evident in all four graphs (note the sign of the 


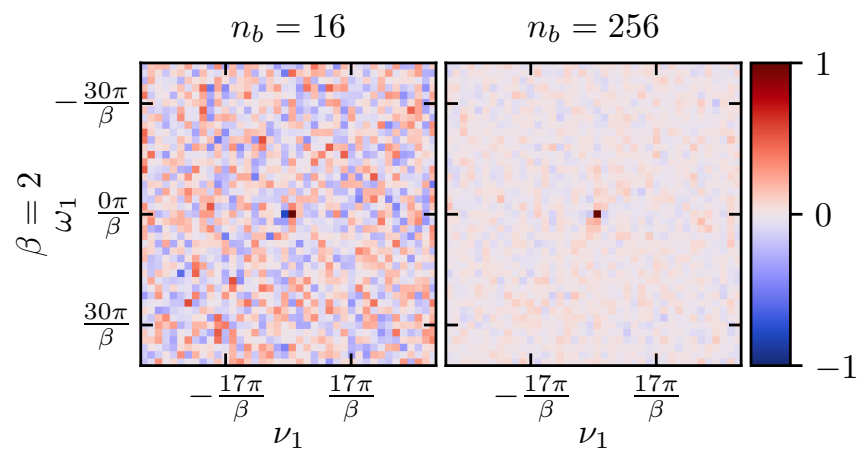

Figure 2. Same as Fig. 1 (top) but now for the estimated correlation matrix $\widehat{\operatorname{corr}}\left[G_{\text {cut2 }}^{(2)}\left(\omega_{1}\right), G_{\text {cut1 }}^{(2)}\left(\nu_{1}\right)\right]$, i.e., the correlation between cut2 with $\omega_{1}, \nu^{\prime}=\nu=0$ and cut1 with $\omega=0$, $\nu^{\prime}=\nu=\nu_{1}$. The $41 \times 40$ correlation matrices correspond to 41 bosonic and 40 fermionic frequencies. As in Fig. 1 for sufficiently many bins (right) the off-diagonal components of the correlation matrix vanish. (Note that in this figure there are no diagonal components, since there are different frequencies on the axes.)

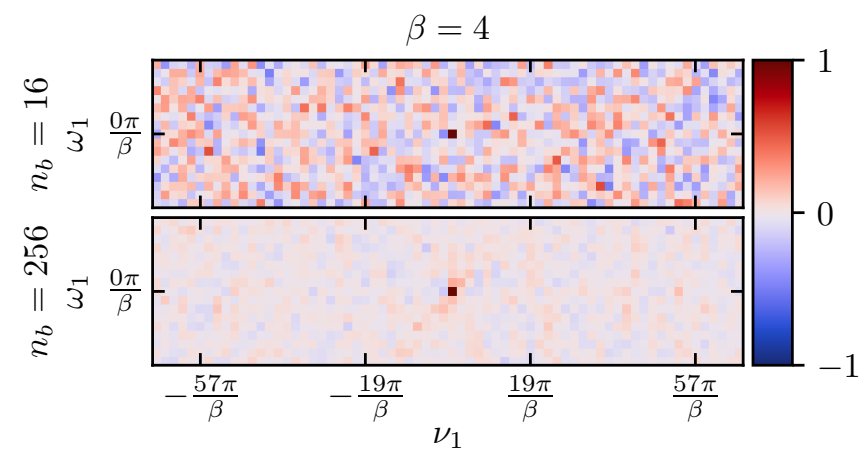

Figure 3. Same as Fig. 2 but now at $\beta=4$. The $17 \times 70$ matrices correspond to 70 fermionic and 17 bosonic frequencies (which we reduced to save computational time as there was no measurable change in the self-energy).

$y$-axis resembling the typical arrangement in a matrix). This suggests that the two-particle Green's function is uncorrelated at different frequencies, at least along the cut.

To further test this, we next consider the estimated correlation between

$$
G_{\text {cut } 2}^{(2)}\left(\omega_{1}\right):=G_{\uparrow \uparrow}^{(2), \nu=0, \nu^{\prime}=0, \omega=\omega_{1}}
$$

and $G_{\text {cut } 1}^{(2)}$ which is shown for $\beta=2$ in Fig. 2 and for $\beta=4$ in Fig. 3 The same numbers of bins are used as before. Apart from the noise, the correlation vanishes again.

Altogether, this suggests that it is reasonable to assume that the two-particle Green's function at different frequencies as calculated with QMC is uncorrelated. Since this is the input to the subsequent DMFT or DГA calculations, any correlations of the output data must emerge through the post-processing.

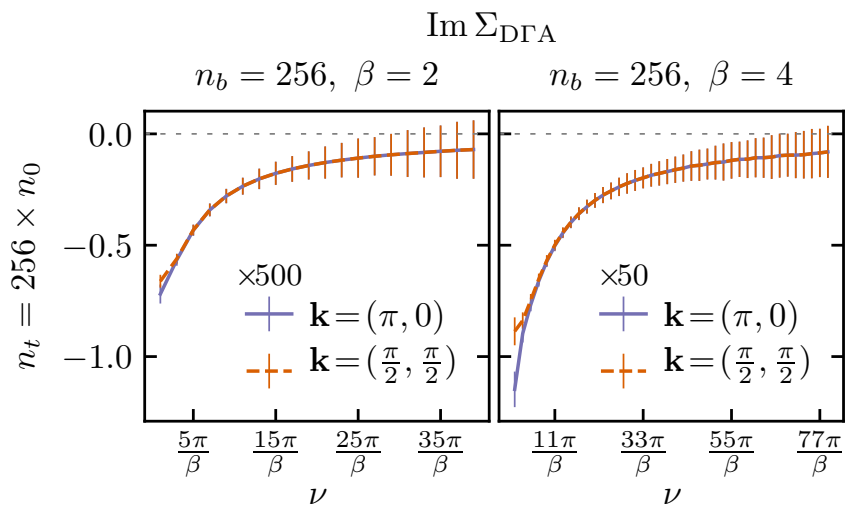

Figure 4. Imaginary part and jackknife SEM of the DГA selfenergy at different temperatures. For better visibility, the errors bars are enlarged by a factor of 500 on the left and by 50 on the right.

\section{SELF-ENERGY}

From the local two-particle Green's function as well as the local one-particle Green's function as an input, the self-energy $\Sigma_{\mathrm{D} \Gamma \mathrm{A}, \nu \mathbf{k}}$ of the $2 \mathrm{D}$ square lattice Hubbard model at half-filling is calculated in DГA. All results in this section are generated using the same parameters as in Section III $U=4 t, \beta=\{2 / t, 4 / t\}, t=1$, $t^{\prime}=0$, i.e., only nearest neighbor hopping is considered. The DГA calculations use nine bosonic as well as 40 and 80 fermionic frequencies for $\beta=2$ and $\beta=4$, respectively. The inner momentum-(k-)grid is $48 \times 48$ and the transfer momentum-(q-) grid is $12 \times 12$ for $\beta=2$ and $24 \times 24$ for $\beta=4$. Before jackknife resampling is applied, the total number $n_{t}$ of QMC measurements of the two-particle Green's function is divided equally into $n_{b}$ bins with $n_{m}=n_{t} / n_{b}$ measurements per bin which are averaged for each bin. These $n_{b}$ averages are then used as the input samples for the jackknife. For a more compact notation and easier comparison of the multi panel figures in this section it is convenient to give the number of measurements as a multiple of $n_{0}=2.4 \times 10^{6}$.

\section{A. Standard error of the mean (SEM)}

Figure 4 shows the imaginary part of the DГА selfenergy at $\beta=2$ and $\beta=4$ using 256 bins with $n_{0}$ QMC measurements each. Note that the error bars are enlarged by a factor of 500 for the left and 50 for the right plot. Taking this rescaling of the error into account, the SEM for $\beta=4$ (right) is about 10 times higher than for $\beta=2$ (left) but still very small. We do not plot the real part of the self-energy because on the Fermi surface at half-filling it is just a constant.

Since ADGA actually calculates two-particle corrections to the DMFT self-energy it is more reasonable to plot the error bars of the difference between the DMFT and DГA self-energy, $\Sigma_{\mathrm{D \Gamma A}, \mathbf{k} \nu}-\Sigma_{\mathrm{DMFT}, \nu}$. In Fig. 5 the 


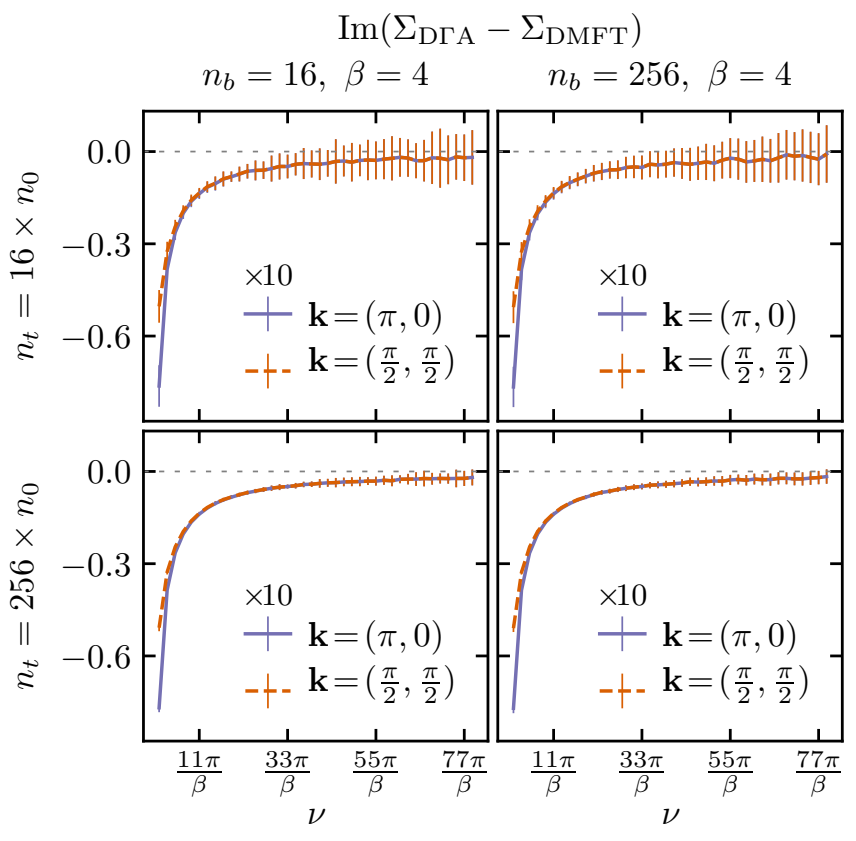

Figure 5. Imaginary part and jackknife SEM of the difference between the DMFT and DГA self-energy, $\Sigma_{\mathrm{D} \Gamma \mathrm{A}, \mathbf{k} \nu}-\Sigma_{\mathrm{DMFT}, \nu}$, at $\beta=4$. Different numbers of bins and total measurements are compared. For better visibility the error bars are enlarged by a factor of 10 .

imaginary part of this DГA self-energy correction is plotted for $\beta=4$ and various combinations of the total number of QMC measurements $n_{t}$ and number of bins $n_{b}$. In the top (bottom) row $n_{t}=16 \times n_{0}\left(256 \times n_{0}\right)$; in the left (right) column $n_{b}=16(256)$ bins. It is obvious that the SEM scales with $n_{t}$. That is, the error for the larger number of measurements $n_{t}$ (bottom row in Fig. 5 is smaller than for a smaller $n_{t}$ (top row in Fig. 5), just as expected. However, there is practically no dependence of the error on the number of bins $n_{b}$ (left vs right column of Fig. 5). One can also see that even only $16 \times n_{0} \approx 4 \times 10^{7}$ total measurements (top row in Fig. 5) lead to still acceptable sizes of the error bars (note they are resized by a factor of ten).

\section{B. Correlation matrix}

Let us now turn to the correlation of the different frequency components of the DГA self-energy. Figure 6 shows the real part of the estimate for the correlation of $\Sigma_{\mathrm{D} \Gamma \mathrm{A}, \mathbf{k} \nu}$ with $\Sigma_{\mathrm{D} \Gamma \mathrm{A}, \mathbf{k} \nu^{\prime}}$ for $\beta=4$ and $\mathbf{k}=(\pi, 0)$. (For the statistical analysis of the input data, we refer the reader to Section [II]) It uses the same layout as Fig. 5 . i.e., $n_{t}=16 \times n_{0}$ in the top row and $n_{t}=256 \times n_{0}$ in the bottom one, with $n_{b}=16$ on the left and $n_{b}=256$ on the right. Contrary to the SEM, the estimated correlation matrix strongly depends on $n_{b}$ as the comparison between the left and right column in Fig. 6 6 shows. While increasing $n_{t}$ does improve the noise slightly, a large num-

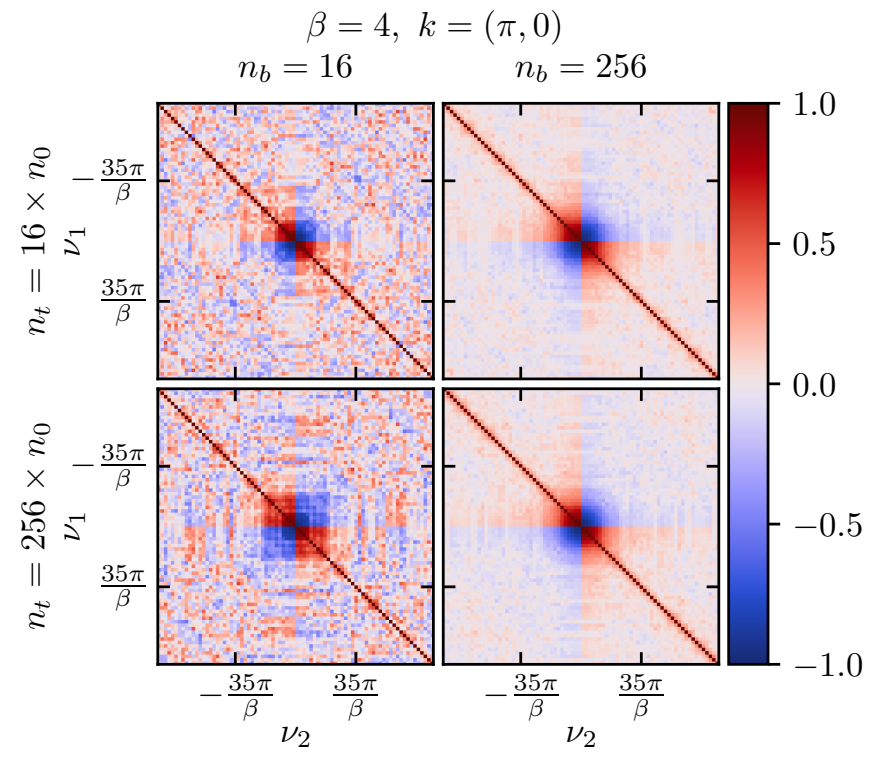

Figure 6. Real part of the estimated correlation matrix $\widehat{\operatorname{corr}}\left[\Sigma_{\mathrm{D} \Gamma \mathrm{A}, \mathbf{k} \nu_{1}}, \Sigma_{\mathrm{D \Gamma \textrm {A } , \mathbf { k } \nu _ { 2 }}}\right]$. As in Fig. 5 different numbers of bins and total measurements are compared. The $80 \times 80$ matrices correspond to 80 fermionic frequencies.

ber of jackknife samples is crucial for an acceptable noise level. It is evident in all four plots that the largest correlations appear in the low-frequency region. Disregarding noise, the only correlations outside of this area are those between low and high frequencies.

Figure 7 shows the dependence of the real part of the estimated correlation matrix on $\beta$ (from the top to the bottom of Fig. 7) and $\mathbf{k}$ (from left to right). One can see that the correlation increases slightly with increasing $\beta$ (or decreasing temperature) and is also more pronounced for $\mathbf{k}=(\pi, 0)$ (right) than for $\mathbf{k}=(0,0)$ (left). However, the general structure of the matrix - high correlation at low frequencies, very low correlation otherwise - is similar in all cases.

We do not plot the imaginary part of the estimated correlation matrix because with the chosen parameters (half-filling and k-points on Fermi surface) it vanishes and shows only noise.

The cross-correlation between frequencies of the DГA self-energy can be understood from Equation (15). It can be rewritten, such that we have the DMFT self-energy plus non-local corrections arising from the two-particle Green's function 22. Both DMFT and DГA self-energy have the same asymptotic behavior, thus non-local corrections have to be restricted to the lower Matsubara frequencies. Since here we consider the correlation arising from the two-particle Green's function, we can conclude that they mainly influence the low-frequency region. Furthermore, in the model under consideration vertex corrections increase with lower temperatures, and the largest influence is to be expected at $\mathbf{k}=(\pi, 0)$, where the formation of a pseudo-gap can be observed.

The symmetries in Figs. 6 and 7 can be explained by 


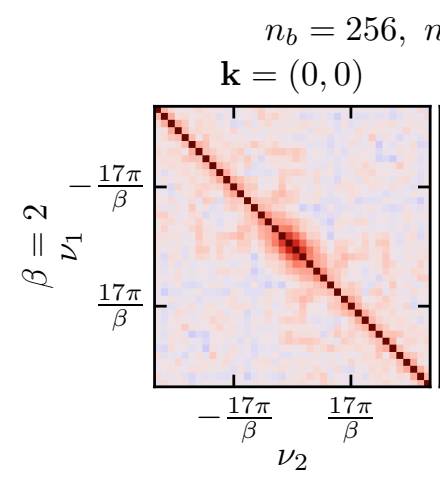

$$
n_{t}=256 \times n_{0}
$$

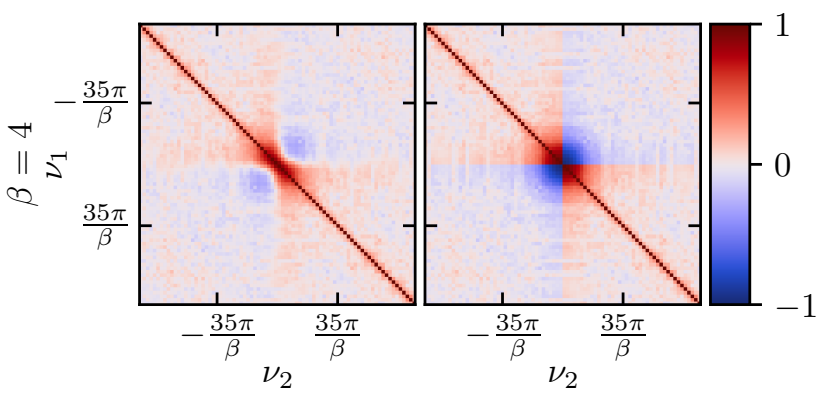

Figure 7. Real part of the estimated correlation matrix $\widehat{\operatorname{corr}}\left[\Sigma_{\mathrm{D \Gamma A}, \mathbf{k} \nu_{1}}, \Sigma_{\mathrm{D \Gamma A}, \mathbf{k} \nu_{2}}\right]$ at different temperatures and $\mathbf{k}-$ points. The $40 \times 40$ (top) and $80 \times 80$ matrices (bottom) correspond to 40 and 80 fermionic frequencies, respectively.

the definition of the estimated correlation coefficients and properties of the self-energy. Using Eq. 27) and $\Sigma(\nu)^{*}=$ $\Sigma(-\nu)$ yields

$$
\begin{aligned}
\widehat{\operatorname{corr}}\left[\Sigma\left(\nu_{1}\right), \Sigma\left(\nu_{2}\right)\right] & =\widehat{\operatorname{corr}}\left[\Sigma\left(\nu_{2}\right), \Sigma\left(\nu_{1}\right)\right]^{*} \\
& =\widehat{\operatorname{corr}}\left[\Sigma\left(\nu_{2}\right)^{*}, \Sigma\left(\nu_{1}\right)^{*}\right] \\
& =\widehat{\operatorname{corr}}\left[\Sigma\left(-\nu_{2}\right), \Sigma\left(-\nu_{1}\right)\right]
\end{aligned}
$$

Therefore the real part of the correlation matrices is symmetric around the main- and antidiagonal.

\section{SUSCEPTIBILITIES}

For periodic systems, the density and magnetic DMFT susceptibilities $\chi_{d}(\mathrm{i} \omega, \mathbf{q})$ and $\chi_{m}(\mathrm{i} \omega, \mathbf{q})$ are the Fourier transform of the following imaginary time expectation values:

$$
\begin{aligned}
\chi_{d}\left(\tau, l-l^{\prime}\right) & =\left\langle T_{\tau}\left(n_{l \uparrow}+n_{l \downarrow}\right)(\tau)\left(n_{l^{\prime} \uparrow}+n_{l^{\prime} \downarrow}\right)(0)\right\rangle, \\
\chi_{m}\left(\tau, l-l^{\prime}\right) & =\left\langle T_{\tau}\left(n_{l \uparrow}-n_{l \downarrow}\right)(\tau)\left(n_{l^{\prime} \uparrow}-n_{l^{\prime} \downarrow}\right)(0)\right\rangle .
\end{aligned}
$$

Here $l$ and $l^{\prime}$ are lattice site indices, $T_{\tau}$ is the timeordering operator and $n_{l \sigma}$ is the electron density at site $l$ with spin $\sigma \in\{\uparrow, \downarrow\}$. In DMFT, they are calculated in momentum space for the square-lattice Hubbard model at half-filling, using the Bethe-Salpeter equations discussed in Section IIA All results in this section are generated using the same parameters as in Section III $U=4 t, \beta=\{2 / t, 4 / t\}$, where $t=1$ sets the energy unit,

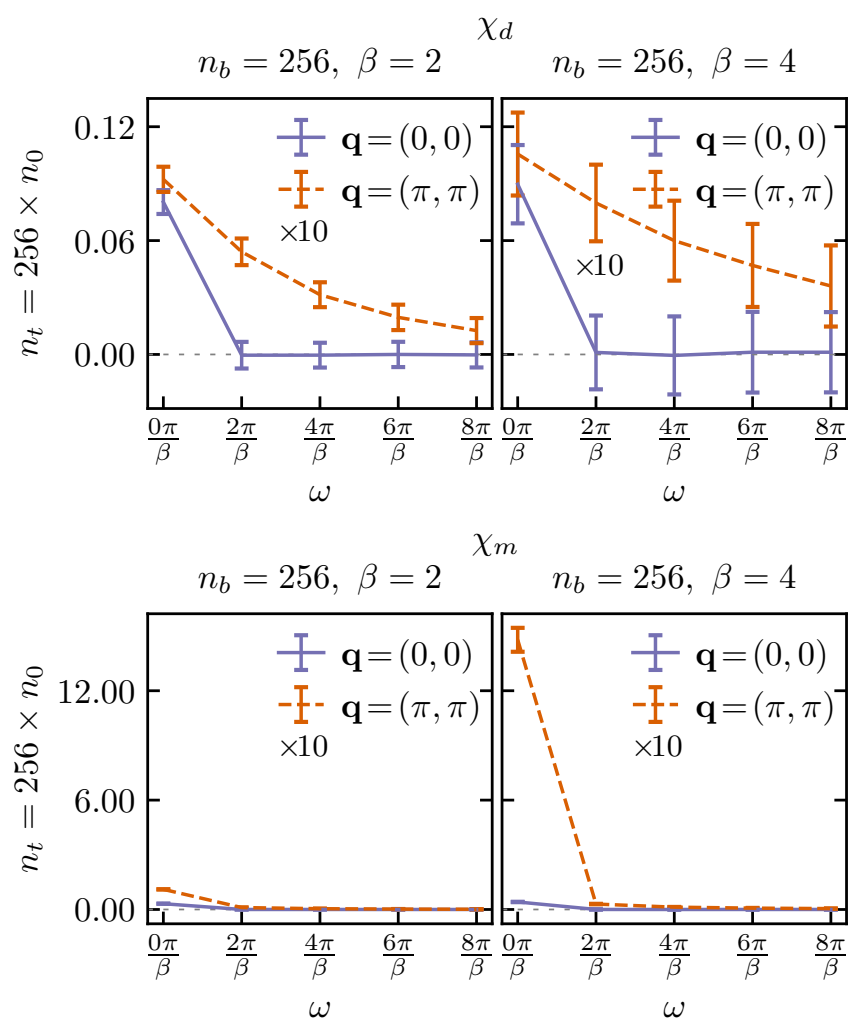

Figure 8. Density and magnetic DMFT susceptibility, $\chi_{d}$ and $\chi_{m}$, at different temperatures and momenta. For better visibility the error bars are enlarged by a factor of 10 .

and only nearest-neighbor hopping is considered. Again, we use nine bosonic as well as 40 and 80 fermionic frequencies for $\beta=2$ and $\beta=4$, respectively. The inner momentum- or k-grid (for the one-particle quantities and $\left.\chi_{0}\right)$ is $48 \times 48$, whereas the transfer momentum- or $\mathbf{q}$ grid is $12 \times 12$ for $\beta=2$ and $24 \times 24$ for $\beta=4$. As before, the total number of QMC measurements $n_{t}$ for the two-particle Green's function is given in multiples of $n_{0}=2.4 \times 10^{6}$ and divided into $n_{b}$ bins, with $n_{m}=n_{t} / n_{b}$ measurements averaged per bin. These averages are then used as the input samples for the jackknife. Note that at half-filling, the susceptibilities and therefore also their correlation matrices are purely real.

\section{A. Standard error of the mean (SEM)}

Figure 8 shows the density and magnetic susceptibilities at $\beta=2$ and $\beta=4$, where $n_{t}=256 \times n_{0}$ measurements are divided into $n_{b}=256$ bins. Since there is a transition to an antiferromagnetic phase at $\beta=4.3$ [33], $\chi_{m}(\mathbf{q}=(\pi, \pi))$ as well as its error are dominated by the contributions at $\omega=0$. This makes it harder to compare the plots of the two susceptibilities but one can still see that the SEM increases with $\beta$ (left vs right panels) for both quantities, just like in the case of the DГA selfenergy. 


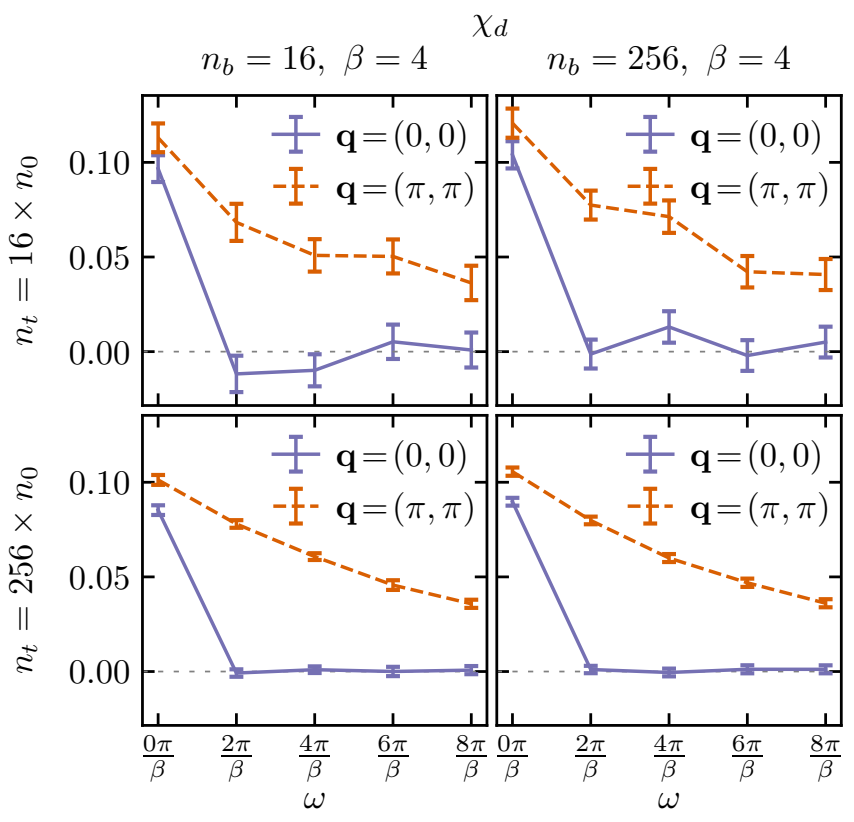

Figure 9. DMFT density susceptibility $\chi_{d}$ at $\beta=4$ for two different momenta $\mathbf{q}$, comparing different numbers of bins and total measurements.

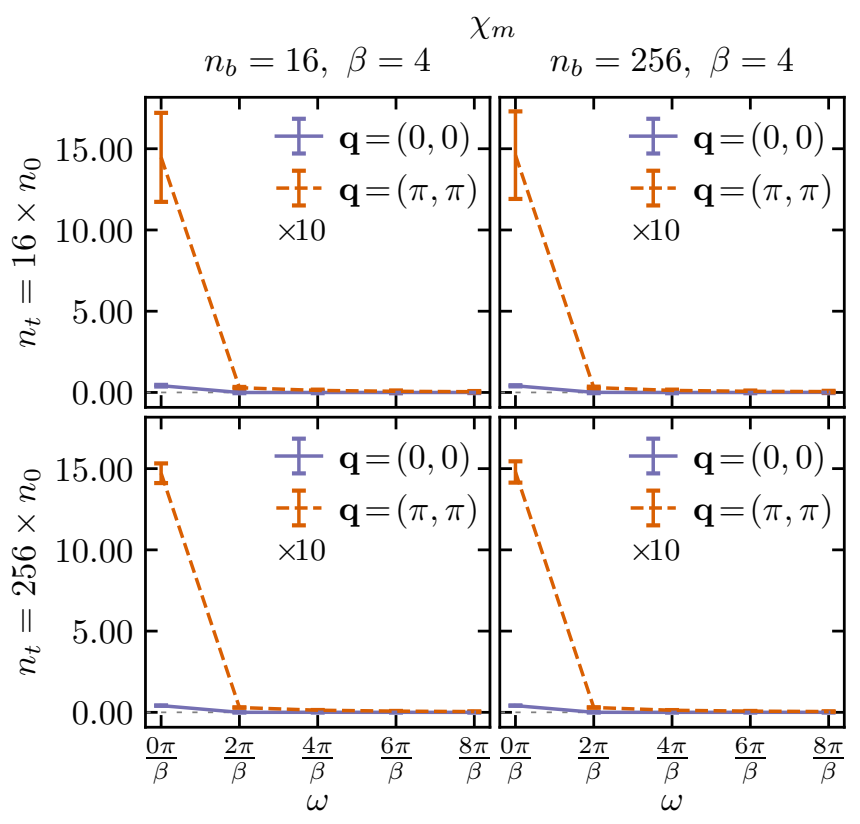

Figure 10. Same as Fig. 9 but for the magnetic susceptibility $\chi_{m}$. For better visibility the error bars are enlarged by a factor of 10 .

The dependence on the total number of measurements $n_{t}$ and the number of bins $n_{b}$ is shown in Fig. 9 for $\chi_{d}$ and in Fig. 10 for $\chi_{m}$; both at $\beta=4$. They use the same layout as Fig. 5 i.e., $n_{t}=16 \times n_{0}$ in the top rows and $256 \times n_{0}$ in the bottom ones, with $n_{b}=16$ on the left and 256 on the right. Only the error bars of $\chi_{m}$ are enlarged by a factor of 10 . Similar to Section IV

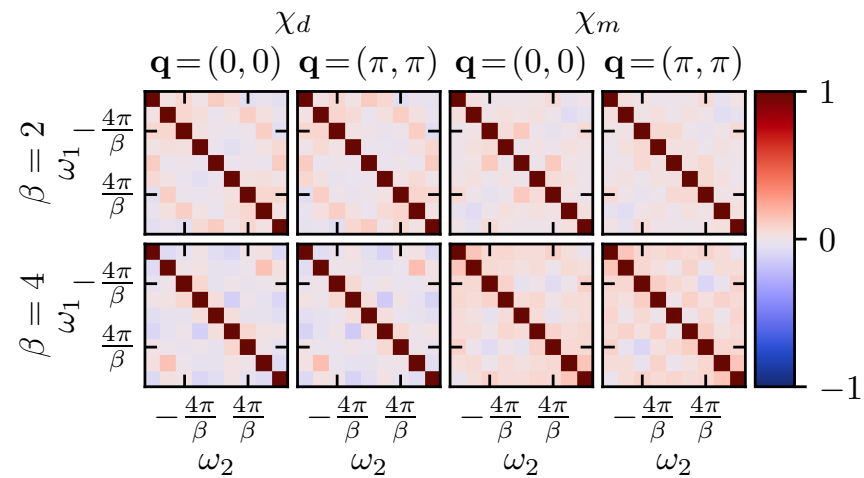

Figure 11. Estimated correlation matrix of the DMFT susceptibilities $\widehat{\operatorname{corr}}\left[\chi_{r}\left(\omega_{1}\right), \chi_{r}\left(\omega_{2}\right)\right], r \in\{m, d\}$ at different temperatures and momenta. A total number of $256 \times n_{0}$ measurements are binned into 256 jackknife samples. The $9 \times 9$ matrices correspond to 9 bosonic frequencies.

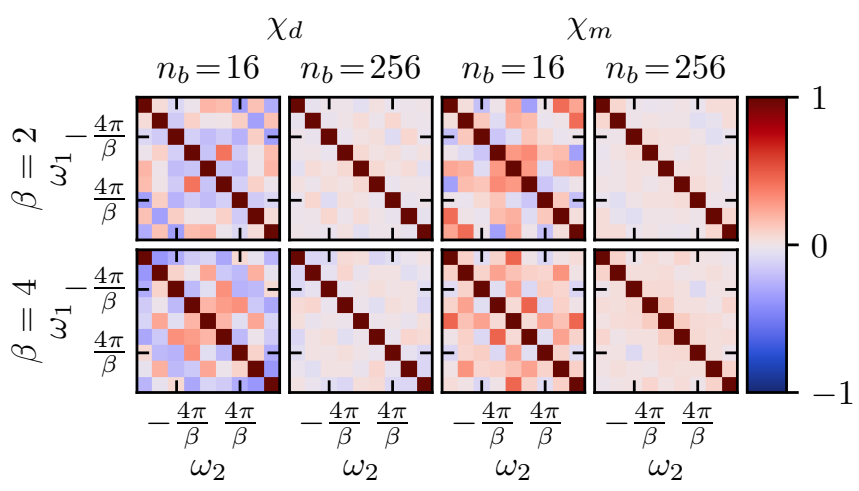

Figure 12. Same as Fig. 11 but now at $\mathbf{q}=(\pi, 0)$ and comparing different numbers of jackknife bins $n_{b}$ for a total number of $16 \times n_{0}$ measurements.

the SEM scales with the total number of measurements (top vs. bottom panels), but it does not depend on the number of jackknife samples $n_{b}$ (left vs. right panels). Contrary to the results of the self-energy, even using a total of $256 \times n_{0} \approx 6 \times 10^{8}$ measurements only yields borderline acceptable error bars. This means the main features of the $\chi_{d}$ curve are still recognizable but larger error bars would render the signal statistically insignificant. Therefore one should aim for at least $O\left(10^{9}\right)$ total measurements in this case.

\section{B. Correlation matrix}

The estimate for the correlation of the susceptibilities with themselves is shown in Fig. 11. Both $\chi_{d}$ and $\chi_{m}$ are uncorrelated for both temperatures $(\beta=2$ and $\beta=4)$ and for both momenta $[\mathbf{q}=(0,0)$ and $\mathbf{q}=(\pi, \pi)]$. The same is true for $\mathbf{q}=(\pi, 0)$, shown in Fig. 12 , which studies the effects of using more jackknife bins. Clearly, a good estimate of the correlation matrix of the susceptibility requires more than $O(10)$ bins. 
The symmetries of the correlation matrices in Figs. 11 and 12 are the same as those in Section IVB. They are symmetric around the main- and antidiagonal because $\chi_{m, d}(\omega)=\chi_{m, d}(-\omega)$, which is the same property as that of the self-energy considering that the susceptibilities are also real.

\section{MAXIMUM ENTROPY ANALYTIC CONTINUATION}

Extracting real-frequency information, $A(\omega)$, out of Matsubara-frequency data, $y(\nu)$, amounts to solving the following fitting problem:

$$
\min _{A}\left\|y(\nu)-\int \mathrm{d} \omega K(\nu, \omega) A(\omega)\right\|=\min _{\mathbf{A}}\|\mathbf{y}-K \mathbf{A}\|,
$$

where $K$ is an integral kernel which is different for bosonic or fermionic functions. $\mathbf{y}$ and $\mathbf{A}$ are the Fourier coefficients of $y(\nu)$ and $A(\omega)$ in an appropriate basis. Equation (35) is minimal if and only if the log-likelihood:

$$
L[\mathbf{A}]=-\frac{1}{2}(\mathbf{y}-K \mathbf{A})^{\dagger} C^{-1}(\mathbf{y}-K \mathbf{A}),
$$

is maximal, where $C$ is the covariance matrix (a positive definite symmetric matrix). Equations (35) and (36) are ill-posed on numerical data as the singular values of $K$ drop super-exponentially.

The maximum entropy method (MEM) [34] is a widely employed method to regularize this problem. Briefly, instead of Eq. (35), we maximize an augmented functional:

$$
Q_{\alpha}[\mathbf{A}]=L[\mathbf{A}]+\alpha S\left[\mathbf{A} \mid \mathbf{A}_{0}\right],
$$

where $S\left[\mathbf{A} \mid \mathbf{A}_{0}\right]$ is the relative (information) entropy with respect to an a priori default model $\mathbf{A}_{0}$. This term regularizes the optimization and has to be scaled by a hyperparameter $\alpha$. Equation (37) can be used on numerical data: $\mathbf{y}$ is now the sample mean and $C$ is the sample covariance matrix in Eq. (36).

Equation 36 can be evaluated much more efficiently, if the covariance matrix is diagonal. However, if that is not the case, we may still perform the transformation $C=\mathcal{U}^{\dagger} V \mathcal{U}$, where $\mathcal{U}$ is unitary and $V$ is a positive definite diagonal matrix. Equation (36) then acquires the simpler form

$$
L[\mathbf{A}]=-\frac{1}{2} \sum_{j} \frac{\left|\tilde{\mathbf{y}}_{j}-(\tilde{K} \mathbf{A})_{j}\right|^{2}}{V_{j}}
$$

with $\tilde{\mathbf{y}}=\mathcal{U} \mathbf{y}$ and $\tilde{K}=\mathcal{U} K$.

If this rotation of the data and the kernel is done as a pre-processing step, then the remaining problem is identical to the case where the covariance matrix is diagonal. In particular, it is still possible to treat real and imaginary part as separate variables by stacking $\tilde{\mathbf{y}} \rightarrow[\operatorname{Re} \tilde{\mathbf{y}}, \operatorname{Im} \tilde{\mathbf{y}}]$ and $\tilde{K} \rightarrow[\operatorname{Re} \tilde{K}, \operatorname{Im} \tilde{K}]$. We use the

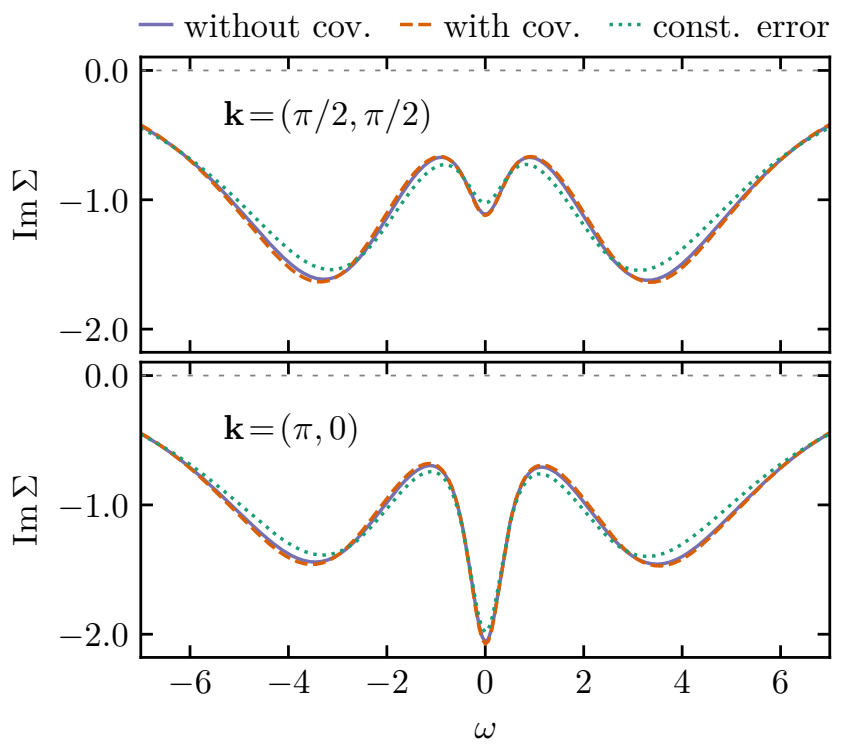

Figure 13. Imaginary part of the self-energy on the realfrequency axis at different momenta $\mathbf{k}$, comparing the analytic continuation without covariance matrix, with the proper covariance matrix, and with a constant error.

ana_cont library 35,36 to perform analytic continuations of the DГA self-energy at $\beta=4$. To this end, we have to subtract the Hartree energy $U / 2$, such that asymptotically also the real part approaches zero. Then the standard kernel for fermionic Green's functions can be used [37] and we obtain a function $A_{\Sigma}(\omega)$ which is related to the imaginary part of the self-energy by

$$
A_{\Sigma}(\omega)=-\frac{1}{\pi} \operatorname{Im} \Sigma\left(\omega+\mathrm{i} 0^{+}\right) .
$$

In Fig. 13 we see that in this case the correlations are not strong enough to make the analytic continuation instable. However, using a constant error rather the one calculated by the jackknife method leads to a slightly different result.

\section{CONCLUSION AND DISCUSSION}

We have implemented and studied a jackknife error estimate for typical DMFT and DГA post-processing calculations after a QMC solution of the Anderson impurity problem. While mere QMC error bars have been analyzed before (also employing the jackknife[38, 39]), the error of the post-processed quantities such as the DMFT susceptibilities and the DГA self-energies has not been systematically studied. Here, the QMC statistical error is propagated through non-linear equations, namely the Bethe-Salpeter equation. In such a situation the jackknife method is the method of choice, and we make our routines that have been tested with QMC input from w2dynamics [20] available at Ref. 40. 
From a statistical point of view, our study reveals that the different components of the two-particle Green's function, which is the CT-HYB QMC output and main jackknife input, are uncorrelated. But a binning into $O(100)$ bins is necessary to remove the spurious off-diagonal components of the correlation matrix. The same holds for the DMFT susceptibility which is calculated through the Bethe-Salpeter equation. Because of the vanishing offdiagonal elements of the correlation (or covariance) matrix, an analytical continuation without covariance is possible.

The DГA self-energy on the other hand has a nondiagonal correlation matrix. Its calculation consists of the Bethe-Salpeter equation, as in DMFT, and additionally the Schwinger-Dyson equation. We conclude that the latter leads to the correlations between the DГA selfenergy at different frequencies. This is also quite intuitive since the same (bosonic) frequency component of the susceptibility contributes to the DГA self-energy at different fermionic frequencies. However, we have shown that the results of analytic continuation of the self-energy are hardly influenced by this. We thus conclude that the correlations introduced by two-particle corrections are still small enough to allow for a reliable interpretation of the results. Still one should keep in mind that using the actual jackknife error, as opposed to a constant, does have a small influence on the analytic continuation.

We have further studied the standard error of the mean (SEM) and observe that it hardly depends on the number of bins $n_{b}$ into which the total number of measurements $n_{t}$ is divided. However as a matter of course the SEM depends strongly on $n_{t}$. Depending on the physical quantity studied and the required accuracy, a total number of measurements $n_{t}$ of $O\left(10^{7}\right)$ to $O\left(10^{9}\right)$ is needed. Here, the error bars of the DMFT susceptibilities are somewhat larger than for the DГA self-energy. The error also increases with decreasing temperature or larger interval $[0, \beta]$, as this interval is sampled less accurately if $n_{t}$ is kept fixed.

Our paper has shown that the jackknife method is a valuable tool for calculating DMFT and DГA error bars. Beyond the present paper, the statistical error of the one-particle Green's function can be included. However, with the use of symmetric improved estimators the errors of the one-particle Green's function are practically nonexistent. A further extension would be to consider the error of the DMFT self-consistency loop itself by drawing bins from statistically independent DMFT solutions. The latter can be achieved either by completely independent DMFT calculations or by determining the autocorrelation time of the DMFT loop and adjusting the binning to it. Our approach can be combined with various other methods to reduce the Monte Carlo noise 27, 29, 41. or the cutoff error of the frequency box 28, 42,45 as well as with compactifications of the vertex [46 48. We hope that our paper will spread the seed for a proper error estimate in future DMFT calculations and diagrammatic extensions thereof.

\section{ACKNOWLEDGMENTS}

The present research was supported by the Austrian Science Fund (FWF) through the Doctoral School W1243 Building Solids for Function (P. K.) as well as projects P32044 and P30997. Calculations were done on the Vienna Scientific Cluster (VSC).
[1] W. Metzner and D. Vollhardt, Phys. Rev. Lett. 62, 324 (1989)

[2] A. Georges and G. Kotliar, Phys. Rev. B 45, 6479 (1992)

[3] M. Jarrell, Phys. Rev. Lett. 69, 168 (1992)

[4] A. Georges, G. Kotliar, W. Krauth, and M. J. Rozenberg, Rev. Mod. Phys. 68, 13 (1996).

[5] V. I. Anisimov, A. I. Poteryaev, M. A. Korotin, A. O. Anokhin, and G. Kotliar, Journal of Physics: Condensed Matter 9, 7359 (1997)

[6] A. I. Lichtenstein and M. I. Katsnelson, Phys. Rev. B 57, 6884 (1998)

[7] K. Held, I. A. Nekrasov, G. Keller, V. Eyert, N. Blümer, A. K. McMahan, R. T. Scalettar, T. Pruschke, V. I. Anisimov, and D. Vollhardt, physica status solidi (b) 243, 2599 (2006), previously appeared as Psi-k Newsletter No. 56 (April 2003).

[8] G. Kotliar, S. Y. Savrasov, K. Haule, V. S. Oudovenko, O. Parcollet, and C. A. Marianetti, Rev. Mod. Phys. 78, 865 (2006)

[9] K. Held, Advances in Physics 56, 829 (2007)

[10] M. Ulmke, V. Janiš, and D. Vollhardt, Phys. Rev. B 51, 10411 (1995)
[11] K. Held, M. Ulmke, N. Blümer, and D. Vollhardt, Phys. Rev. B 56, 14469 (1997).

[12] M. H. Hettler, M. Mukherjee, M. Jarrell, and H. R. Krishnamurthy, Phys. Rev. B 61, 12739 (2000).

[13] G. Rohringer, H. Hafermann, A. Toschi, A. A. Katanin, A. E. Antipov, M. I. Katsnelson, A. I. Lichtenstein, A. N. Rubtsov, and K. Held, Rev. Mod. Phys. 90, 025003 (2018)

[14] A. Toschi, A. A. Katanin, and K. Held, Phys Rev. B 75, 045118 (2007).

[15] A. A. Katanin, A. Toschi, and K. Held, Phys. Rev. B 80, 075104 (2009)

[16] T. Ribic, P. Gunacker, S. Iskakov, M. Wallerberger, G. Rohringer, A. N. Rubtsov, E. Gull, and K. Held, Phys. Rev. B 96, 235127 (2017).

[17] A. N. Rubtsov, M. I. Katsnelson, and A. I. Lichtenstein, Phys. Rev. B 77, 033101 (2008)

[18] R. G. Miller, Ann. Math. Statist. 35, 1594 (1964)

[19] R. G. Miller, Biometrika 61, 1 (1974)

[20] M. Wallerberger, A. Hausoel, P. Gunacker, A. Kowalski, N. Parragh, F. Goth, K. Held, and G. Sangiovanni, Computer Physics Communications 235, 388 (2019). 
[21] E. Gull, A. J. Millis, A. I. Lichtenstein, A. N. Rubtsov, M. Troyer, and P. Werner, Rev. Mod. Phys. 83, 349 (2011)

[22] A. Galler, P. Thunström, P. Gunacker, J. M. Tomczak, and K. Held, Phys. Rev. B 95, 115107 (2017)

[23] A. Galler, P. Thunström, J. Kaufmann, M. Pickem, J. M. Tomczak, and K. Held, Computer Physics Communications 245, 106847 (2019)

[24] P. Werner, A. Comanac, L. de' Medici, M. Troyer, and A. J. Millis, Phys. Rev. Lett. 97, 076405 (2006).

[25] P. Gunacker, M. Wallerberger, E. Gull, A. Hausoel, G. Sangiovanni, and K. Held, Phys. Rev. B 92, 155102 (2015)

[26] N. Parragh, A. Toschi, K. Held, and G. Sangiovanni, Phys. Rev. B 86, 155158 (2012)

[27] P. Gunacker, M. Wallerberger, T. Ribic, A. Hausoel, G. Sangiovanni, and K. Held, Phys. Rev. B 94, 125153 (2016)

[28] J. Kaufmann, P. Gunacker, and K. Held, Phys. Rev. B 96, 035114 (2017).

[29] J. Kaufmann, P. Gunacker, A. Kowalski, G. Sangiovanni, and K. Held, Phys. Rev. B 100, 075119 (2019)

[30] B. Efron, The Jackknife, the Bootstrap and Other Resampling Plans (Society for Industrial and Applied Mathematics, Philadelphia, Pennsylvania, 1982).

[31] B. Efron, Ann. Statist. 7, 1 (1979)

[32] R. Wilcox, Fundamentals of Modern Statistical Methods: Substantially Improving Power and Accuracy (Springer New York, 2010).

[33] J. Kuneš, Phys. Rev. B 83, 085102 (2011).
[34] M. Jarrell and J. E. Gubernatis, Physics Reports 269, 133 (1996)

[35] D. Geffroy, J. Kaufmann, A. Hariki, P. Gunacker, A. Hausoel, and J. Kuneš, Phys. Rev. Lett. 122, 127601 (2019)

[36] J. Kaufmann, Ana-cont, https://github.com/ josefkaufmann/ana_cont.git (2019).

[37] J. M. Luttinger, Phys. Rev. 121, 942 (1961).

[38] E. Pavarini, E. Koch, D. Vollhardt, and A. Lichtenstein, DMFT at 25: Infinite Dimensions, Reihe Modeling and Simulation 4, Vol. 4 (Forschungszentrum Jülich Zentralbibliothek, Verlag (Jülich), Jülich, 2014).

[39] M. Bercx, F. Goth, J. S. Hofmann, and F. F. Assaad, SciPost Phys. 3, 013 (2017),

[40] P. Kappl, jackknife, https://gitlab.com/ PatrickKappl/jackknife (2019)

[41] H. Hafermann, K. R. Patton, and P. Werner, Phys. Rev. B 85, 205106 (2012)

[42] N. Wentzell, G. Li, A. Tagliavini, C. Taranto, G. Rohringer, K. Held, A. Toschi, and S. Andergassen, Phys. Rev. B 102, 085106 (2020).

[43] M. Kitatani, T. Schäfer, H. Aoki, and K. Held, Phys. Rev. B 99, 041115 (2019).

[44] F. Krien, Phys. Rev. B 99, 235106 (2019).

[45] A. Katanin, Phys. Rev. B 101, 035110 (2020).

[46] L. Boehnke, H. Hafermann, M. Ferrero, F. Lechermann, and O. Parcollet, Phys. Rev. B 84, 075145 (2011).

[47] H. Shinaoka, J. Otsuki, K. Haule, M. Wallerberger, E. Gull, K. Yoshimi, and M. Ohzeki, Phys. Rev. B 97, 205111 (2018)

[48] H. Shinaoka, D. Geffroy, M. Wallerberger, J. Otsuki, K. Yoshimi, E. Gull, and J. Kuneš, SciPost Phys. 8, 12 (2020). 